\title{
Double Batched DNA Sequencing is a reliable, cost- effective and scalable approach to genomic population screening
}

Ulrik Stoltze ( $\sim$ ulrik.kristoffer.stoltze@regionh.dk)

Rigshospitalet

Christian Hagen

Statens Serum Institut

Thomas Hansen

Rigshospitalet

Anna Byrjalsen

Rigshospitalet

Anne-Marie Gerdes

Rigshospital https://orcid.org/0000-0002-0328-3320

Victor Yakimov

Statens Serum Institut

Simon Rasmussen

University of Copenhagen https://orcid.org/0000-0001-6323-9041

Marie Bækvad-Hansen

Statens Serum Institut

David Hougaard

Statens Serum Institut https://orcid.org/0000-0001-5928-3517

Kjeld Schmiegelow

the University of Copenhagen

Henrik Hjalgrim

Danish Cancer Society Research Centre

Karin Wadt

Rigshospitalet https://orcid.org/0000-0003-2882-6798

Jonas Bybjerg-Grauholm

iPSYCH https://orcid.org/0000-0003-1705-4008

Article

Keywords: 
Posted Date: March 8th, 2022

DOI: https://doi.org/10.21203/rs.3.rs-1407847/v1

License: (c) (i) This work is licensed under a Creative Commons Attribution 4.0 International License. Read Full License 


\section{Abstract}

Next-generation sequencing (NGS) based population screening holds great promise for disease prevention and earlier diagnosis, but associated sequencing costs remain prohibitive. We developed double batched sequencing (DoBSeq) and tested it on neonatal blood spot DNA in an explorative $(\mathrm{n}=$ $100)$ and a validation $(n=100)$ cohort selected from a nation-wide childhood cancer whole genome sequencing (WGS) study. Each cohort was enriched for loss-of-function/pathogenic variants in cancer predisposition syndrome genes. Using a commercial 113-gene panel benchmarked against individual WGS data, we demonstrated replicable detection of deleterious and pathogenic variants, with positive and negative predictive values of $100 \%(95 \% \mathrm{Cl} 0.91-1.00 \& 95 \% \mathrm{Cl} 0.98-1.00$, respectively). Pathogenic variants were detected in RB1, TP53, BRCA2, APC, and 19 other genes. Analyses of larger batches $(\mathrm{n}=24$, $48,72 \& 96)$ indicate that DoBSeq is highly scalable and thus a promising, cost-effective method for realizing the potential of population-scale NGS-based screening.

\section{Introduction}

Rare diseases (RD) are defined as diseases that affect fewer than one in 1,000 to one in 2,000 individuals ${ }^{1}$. More than 6,000 different types of RD have been identified, and it is estimated that as much as one in 16 individuals under the age of 25 years suffers from some form of $\mathrm{RD}^{1}$, corresponding to a quarter of a billion people globally. RDs cause substantial mortality, morbidity, disability and financial burden, in fact, in high-income nations, RDs are arguably the leading cause of childhood mortality, responsible for one in three deaths in children².

Treatment is available for many types of RDs that would reduce associated morbidities if the condition were diagnosed well in advance of its clinical presentation. Currently, however, RD screening is generally limited to the small subset of conditions with established biochemical signatures that can be implied by mass spectroscopy or other techniques ${ }^{3,4}$ and subsequently confirmed with secondary - often genetic tests.

Genetic etiologies have been established for more than $70 \%$ of $\mathrm{RDs}^{1}$, rendering them obvious candidates for genetic screening. Indeed, next-generation DNA sequencing (NGS) technologies are almost all capable of detecting the pathogenic germline variants that cause RDs.

However, various technical challenges notwithstanding ${ }^{5,6}$ the mere costs of NGS has effectively prevented its implementation into large-scale screening ${ }^{7}$. Whether undertaken as single gene, gene-panel or whole exome/genome sequencing (WES/WGS), individual sequencing would be costly for any population-based initiative. Batching of DNA samples has been suggested as a means to reduce NGS costs; however, low reliability has led to this approach being mostly abandoned ${ }^{8}$.

We therefore tested a novel approach for mass genetic screening aimed at overcoming these challenges. We hypothesized that by double batching DNA from all individuals in a population, we could dramatically 
lower sequencing costs, while maintaining high reliability and, most importantly, still be able to immediately assign identified rare variants to specific individuals. The Double Batched sequencing (DoBSeq) approach has been described and investigated previously ${ }^{8,9}$, but without proper benchmarking and parameter validation its performance for real-world applications remains wholly unexplored.

We selected participants from a prospective, nation-wide genomic study of childhood cancer ${ }^{10}$; a disease area that stands to benefit from NGS-based neonatal screening. Highly penetrant cancer predisposition syndromes (CPS) caused by pathogenic germline variants are common in childhood cancer patients ${ }^{10-12}$ and are often clinically silent or discrete prior to the cancer diagnosis, likely contributing to widespread underdiagnosis $^{13,14}$. Furthermore, pediatric CPS variants are frequently de novo, so the only option for pre-symptomatic diagnosis is screening.

In this proof-of-principle study, we aimed to demonstrate that we could reliably and replicably identify CPS-causing deleterious and/or pathogenic variants using DoBSeq on DNA from neonatal blood spots all taken prior to the cancer diagnosis.

\section{Results}

\section{Explorative cohort}

We selected an explorative cohort (EC) of 100 participants, enriched for germline loss-of-function/known pathogenic (LoF/P) variants, among childhood cancer patients with WGS data available. WGS identified 31 high-confidence LoF/P variants [52\% frameshift, $29 \%$ nonsense, $13 \%$ missense and $6 \%$ splice] that were both unique to the cohort and covered by a commercial 113-gene panel (Figure 1, Supplemental Table 1 \& 3).

From each of the 100 EC participants, DNA was extracted from neonatal blood spots and split in two aliquots. Each of the two aliquots was then batched with DNA aliquots from nine other participants, according to a 10 by 10 matrix of all EC participants (Supplemental Figure $1 \& 2$ ). In this way, DNA aliquots from two participants were never batched together more than once, and each participant was therefore represented in a unique batch combination (UBC) of one column and one row in the matrix (Figure 1A, 1B, 1C).

The 113-gene panel was employed for sequencing of all 20 batches. Using WGS data to determine true variants, we optimized a bioinformatic approach for pin-pointing variants from the gene panel to specific individuals in the matrix by cross-referencing unique variants in row and column batches. Final filtering parameters were determined empirically (Supplemental Figure $\mathbf{4} \& \mathbf{5}$ ) and identified 575 cohort-unique variants in the EC of which 35 were LoF/P (Supplemental Figure 6 \& Supplemental Table 3). Of these, $89 \%$ were classified as being either high (27) or medium (4) confidence, and were considered positive, while the remaining $11 \%$ were classified as low (2) or no (2) confidence and were considered negative (Figure 1C). 
The $31 \mathrm{LoF} / \mathrm{P}$ variants called by DoBSeq had complete (100\%) patient-specific overlap with the $31 \mathrm{LoF} / \mathrm{P}$ variants identified by the gold standard WGS data. Thus, using the filtering and confidence-scoring parameters determined in the EC analysis, DoBSeq showed 100\% (CI95\% 0.89-1.00) sensitivity and 100\% (CI95\% 0.95-1.00) specificity for the identification of cohort-unique LoF/P variants found by individual WGS (Figure 1D).

\section{Validation cohort}

Next, we tested replicability in a validation cohort (VC) of 100 patients again selected from the childhood cancer cohort, but with no patients overlapping with the EC. Employing identical methods, we undertook variant calling using only the exact parameters determined in the EC analysis (Supplemental Figure 5). All investigators remained blind to both patient identity and individual WGS data until the VC analysis was complete and LoF/P variants were reported (Figure 1E, 1F \& 1G).

Using only the predetermined parameters, DoBSeq called 537 cohort-unique variants in the VC of which 9 were LoF/P (Supplemental Figure 7 \& Supplemental Table 4). Seven variants were of high (6) or medium (1) confidence and were considered positive, while two were of no confidence and were considered negative (Figure 1G). The seven LoF/P variants that were positive on DoBSeq were reported to unblinded investigators and compared to LoF/P variants found by individual WGS data. Again, all 7 LoF/P variants found using DoBSeq had complete (100\%), patient-specific overlap with the 7 LoF/P variants identified in the WGS data [ $43 \%$ frameshift, $29 \%$ nonsense, $14 \%$ missense and $14 \%$ splice]. Thus, DoBSeq maintained $100 \%$ (CI95\% 0.59-1.00) sensitivity and 100\% (CI95\% 0.96-1.00) specificity for the identification of cohortunique LoF/P variants found with individual WGS (Figure 1H).

Combining results from both the explorative and the validation cohorts yielded a positive predictive value of having a cohort-unique LoF/P variant found on DoBSeq of $100 \%$ (CI95\% 0.91-1.00) and negative predictive value of $100 \%$ (CI95\% 0.98-1.00).

Focusing on TP53, a single gene of particular interest, we reidentified all 9 cohort-unique variants of any classification across both cohorts including two variants [p.lle254Thr and p.Leu257GIn] not reported as pathogenic in ClinVar, but immediately classified as such due to the location in the DNA binding domain and other phenotype or functional data. The validation cohort only revealed the anticipated nonpathogenic variants (Figure 2).

\section{Scalability}

Finally, we tested the sensitivity for detection of LoF/P variants when sequencing stand-alone batches of neonatal blood spot DNA including 24, 48, 72, and 96 individuals from the EC (Figure 3B, Supplemental figure 3 ). The total coverage $(X)$ per batch was kept constant, resulting in coverages per sample that were proportionally lower than the 200X achieved in the EC and VC. Even at an average of 10X per allele (in the 96-sample stand-alone batch), 31 of $31 \mathrm{LoF} / \mathrm{P}$ variants were detected, resulting in a sensitivity that 
remained 100\% (Figure 3A, Supplemental Table 2). However, due to a combination of lower sequencing yield and lower contribution of each allele, false variants became increasingly difficult to distinguish from those known to be true (Supplemental Figure 9).

\section{Discussion}

We demonstrate that the DoBSeq approach to mass genetic screening for rare disease-causing variants is a reliable and cost-effective method, which is likely to be highly scalable and hence applicable to population screening. The high performance and ability to directly pinpoint carriers of genetic variants are a product of the repeated sequencing of the same individual in two separate batches. To eliminate interpretation biases in the present analysis, we purposefully excluded variants that were internally classified as pathogenic (examples in Figure 2).

Surprisingly, our data showed that false positive LoF/P variants with passable quality parameters were widespread when the sequencing results from stand-alone batches were assessed in isolation (Figure 1A, 1B, 1E \& 1F). However, by filtering variants to those seen in a combination of one column and one row batch, i.e., appearing twice in two different batches, could we distinguish between true and false positive variants. This suggests that single batched sequencing (where carriers cannot be pinpointed) would require copious individual resequencing, often in the pursuit of ultimately false positive variants, rendering the approach untenable. Moreover, considering the high number of RDs in the general population, even the pursuit of true positive variants could become costly if dozens or even hundreds of genes were screened simultaneously and each finding required resequencing of each individual in the singleton batch.

Currently, well over 400 gene-disease pairs are considered highly actionable in childhood with respect to age-of-onset and/or timing of intervention, and at least an additional 25 are highly actionable in adulthood $^{15}$. Each of these conditions may be considered viable candidates for population screening, yet, primarily due to cost restrictions, few are routinely screened for in any healthcare system and no screening currently uses genomics up-front ${ }^{7,16}$.

A recent simulation model evaluated the cost-effectiveness of universal screening for a panel including 11 pediatric CPS genes ${ }^{17}$, which fully overlapped with the 113-gene panel used in our study. Because RDs, such as pediatric CPSs, by definition have very low prevalence, the number-needed-to-diagnose (NND) in an unselected cohort is high. For individual rare conditions, such as WT1-related disorders, the price per diagnosis (PPD) at the estimated cost of doing a single genetic test likely exceeds $\$ 10$ million (Figure $3 \mathrm{C}$ ). Of course, by using panels, several conditions can be screened for simultaneously, lowering the NND for any one condition. For the 11 pediatric CPS genes, Yeh et al. ${ }^{17}$ conclude that at a price per sample (PPS) of $\$ 55$, tumor surveillance strategies are cost-prohibitive ( $\$ 244,860$ per life-year gained), yet, at a PPS of $\$ 20$, such strategies approach liberal cost-effectiveness $(<\$ 100,000$ per life-year gained) (Figure 3D). Practically, the PPD must be added to the isolated cost of the tumor surveillance strategy, making it increasingly likely that any given treatment, which is cost-effective in isolation, will remain cost- 
effective as PPD is reduced. According to the models developed by Yeh et al., the tumor surveillance strategies and treatments available for the 11 conditions they studied will be even conservatively costeffective ( $<50,000$ per life-year gained) if PPS drops below $\$ 8$, which we estimate is possible when DoBSeq is run at scale (Figure $3 \mathbf{D}$ ).

DoBSeq has the potential to advance the rate of presymptomatic diagnosis, and the cost-effectiveness of doing so, for hundreds of actionable RDs caused by rare genetic variants. This is by no means limited to DNA samples from neonatal blood spots. Several studies have investigated the cost-effectiveness of population screening for adult CPSs ${ }^{18-22}$. One such study ${ }^{22}$, investigating the impact of PPS on universal adult screening for $B R C A 1 / 2$ and MMR genes, found it to be conservatively cost-effective even at PPS exceeding $\$ 1000$. Lowering PPS to those estimated for DoBSeq running at scale could lead to prices per life-year gained that approach cost-saving (Supplemental Figure 10 \& 11). Most adult CPSs are believed to be undiagnosed, and therefore population based cancer screening stands to improve with genetically informed precision prevention ${ }^{21}$.

The promising aspects of DoBSeq must be viewed in light of some limitations of the method and of this study. If multiple individuals in the same DoBSeq matrix carry exactly the same variant it may not be possible to pinpoint the carriers directly. In this event, however, the number of possible carriers will be limited to a small group amenable for individual (re)sequencing. For instance, if two individuals carried the same variant, (re)sequencing of four individuals would be required to identify the two true carriers. Still, genetic heterogeneity, meaning that a specific disease may be caused by a myriad of distinct genetic defects, generally makes multiple carriers in that same matrix unlikely. This was also found in our study, e.g. for TP53 where four distinct variants all caused the same condition (Li-Fraumeni Syndrome) which is associated with a high risk of childhood cancers ${ }^{23}$ (Figure 2). Another possible limitation was that prior to batching, our method harmonized DNA concentration across all samples, which added a minor cost for each sample. At scale this may impact overall cost-effectiveness estimates and we did not test whether removing this step impacted the performance of DoBSeq. Lastly, structural variants, known to cause around $10 \%$ of $\mathrm{RD}^{24}$, were not investigated as part of the present study.

Lately, combinatorial pooling strategies, like DoBSeq, have received increased attention as a theoretical alternative to the extensive and costly population screening for the SARS-Cov- 2 virus ${ }^{25}$. While the methods appear similar, a crucial difference is that, a disease may be caused by a myriad of distinct genetic variants, whereas SARS-Cov-2 tests, by and large, are positive in the same way.

Finally, we would be remiss not to mention that the implementation of mass genetic screening of healthy neonates, children, and/or adults, precipitates critical ethical considerations ${ }^{26}$. Ethics is not a focus of our current study, however, it is important to note that our method detects rare variants only, thus limiting the amount of personal data obtained per individual. Moreover, if our method is developed further, the low 
price per sample may extend accessibility in low- and middle-income countries, making it more equitable than individual NGS based methods.

In conclusion, the DoBSeq method reliably detects rare pathogenic germline variants in populations at single nucleotide resolution. The approach is highly scalable and may easily be incorporated into existing screening infrastructures based on the collection of blood spot samples. Consequently, DoBSeq may pave the way for cost-effective, at-scale, NGS-based population screening, and studies of large, unselected cohorts using DoBSeq are warranted.

\section{Declarations}

Funding: This study received financial support from the Danish Childhood Cancer Foundation, the Danish Childhood Brain Tumour Foundation, the Danish Cancer Society and the European Union's Interregional Oresund-Kattegat-Skagerrak grant.

Conflicts of interest: None to declare.

Authorship: Conception and design of "STAGING": KS, KW, UKS, AMG. Methodology Idea: JBG, UKS, HH, $\mathrm{CMH}$. Design of current study: UKS, JBG, HH, VY, SR, KS, KW, CMH, TVOH. Patient inclusion and collection of samples: UKS, AB, MABH, DH, JBG. Analysis and interpretation of data: UKS, CMH, JBG, SR, KW, TVOH . Manuscript preparation: UKS, CMH, JBG. Critical revision and approval of Manuscript: All authors

\section{References}

1. Ferreira, C. R. The burden of rare diseases. Am. J. Med. Genet. A. 179, 885-892 (2019).

2. Stevenson, D. A. \& Carey, J. C. Contribution of malformations and genetic disorders to mortality in a children's hospital. Am. J. Med. Genet. A. 126A, 393-397 (2004).

3. Coene, K. L. M. et al. Next-generation metabolic screening: targeted and untargeted metabolomics for the diagnosis of inborn errors of metabolism in individual patients. J. Inherit. Metab. Dis. 41, 337353 (2018).

4. Rare Diseases Epidemiology: Update and Overview. vol. 1031 (Springer International Publishing, 2017).

5. Adams, M. C., Evans, J. P., Henderson, G. E. \& Berg, J. S. The promise and peril of genomic screening in the general population. Genet. Med. Off. J. Am. Coll. Med. Genet. 18, 593-599 (2016).

6. Boemer, F. et al. A next-generation newborn screening pilot study: NGS on dried blood spots detects causal mutations in patients with inherited metabolic diseases. Sci. Rep. 7, 17641 (2017). 
7. Newborn screening: a review of history, recent advancements, and future perspectives in the era of next generation sequencing. https://oce-ovid-com.ep.fjernadgang.kb.dk/article/00008480201612000-00004/HTML.

8. Zuzarte, P. C. et al. A Two-Dimensional Pooling Strategy for Rare Variant Detection on NextGeneration Sequencing Platforms. PLoS ONE 9, e93455 (2014).

9. Bonachea, E. M. et al. Use of a targeted, combinatorial next-generation sequencing approach for the study of bicuspid aortic valve. BMC Med. Genomics 7, 56 (2014).

10. Byrjalsen, A. et al. Nationwide germline whole genome sequencing of 198 consecutive pediatric cancer patients reveals a high incidence of cancer prone syndromes. PLoS Genet. 16, (2020).

11. Zhang, J. et al. Germline Mutations in Predisposition Genes in Pediatric Cancer. N. Engl. J. Med. 373, 2336-2346 (2015).

12. Gröbner, S. N. et al. The landscape of genomic alterations across childhood cancers. Nature $\mathbf{5 5 5}$, 321-327 (2018).

13. Stoltze, U. et al. Clinical characteristics and registry-validated extended pedigrees of germline TP53 mutation carriers in Denmark. PLoS ONE 13, (2018).

14. Beitsch, P. D. et al. Underdiagnosis of Hereditary Breast Cancer: Are Genetic Testing Guidelines a Tool or an Obstacle? J. Clin. Oncol. Off. J. Am. Soc. Clin. Oncol. 37, 453-460 (2019).

15. Milko, L. V. et al. An Age-Based Framework for Evaluating Genome-Scale Sequencing Results in Newborn Screening. J. Pediatr. 209, 68-76 (2019).

16. Loeber, J. G. et al. Neonatal Screening in Europe Revisited: An ISNS Perspective on the Current State and Developments Since 2010. Int. J. Neonatal Screen. 7, 15 (2021).

17. Yeh, J. M. et al. Universal newborn genetic screening for pediatric cancer predisposition syndromes: model-based insights. Genet. Med. (2021) doi:10.1038/s41436-021-01124-x.

18. Sun, L. et al. A Cost-effectiveness Analysis of Multigene Testing for All Patients With Breast Cancer. JAMA Oncol. (2019) doi:10.1001/jamaoncol.2019.3323.

19. Michaan, N. et al. Cost Effectiveness of Whole Population BRCA Genetic Screening for Cancer Prevention in Israel. Cancer Prev. Res. Phila. Pa 14, 455-462 (2021).

20. Meshkani, Z., Aboutorabi, A., Moradi, N., Langarizadeh, M. \& Motlagh, A. G. Population or family history based BRCA gene tests of breast cancer? A systematic review of economic evaluations. Hered. Cancer Clin. Pract. 19, 35 (2021).

21. Evans, O. \& Manchanda, R. Population-based Genetic Testing for Precision Prevention. Cancer Prev. Res. Phila. Pa 13, 643-648 (2020).

22. Zhang, L. et al. Population genomic screening of all young adults in a health-care system: a costeffectiveness analysis. Genet. Med. 21, 1958-1968 (2019).

23. Bougeard, G. et al. Revisiting Li-Fraumeni Syndrome From TP53 Mutation Carriers. J. Clin. Oncol. 33, 2345-2352 (2015). 
24. Sharo, A. G., Hu, Z., Sunyaev, S. R. \& Brenner, S. E. StrVCTVRE: A supervised learning method to predict the pathogenicity of human genome structural variants. Am. J. Hum. Genet. 109, 195-209 (2022).

25. Furstenau, T. N., Cocking, J. H., Hepp, C. M. \& Fofanov, V. Y. Sample pooling methods for efficient pathogen screening: Practical implications. PLoS ONE 15, e0236849 (2020).

26. Downie, L., Halliday, J., Lewis, S. \& Amor, D. J. Principles of Genomic Newborn Screening Programs: A Systematic Review. JAMA Netw. Open 4, e2114336 (2021).

\section{Figures}

Explorative cohort - unblinded (Highly enriched for LoF/P variants)

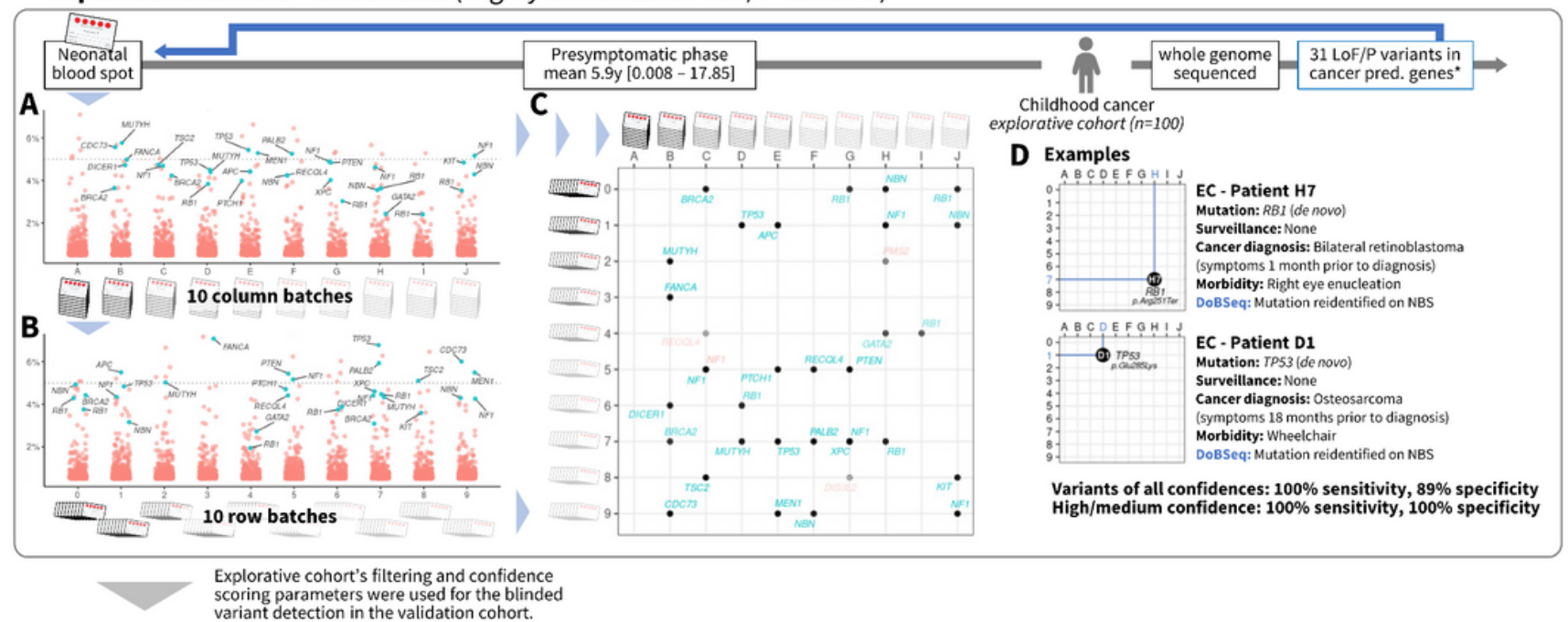

Validation cohort - blinded (Moderately enriched for LoF/P variants)

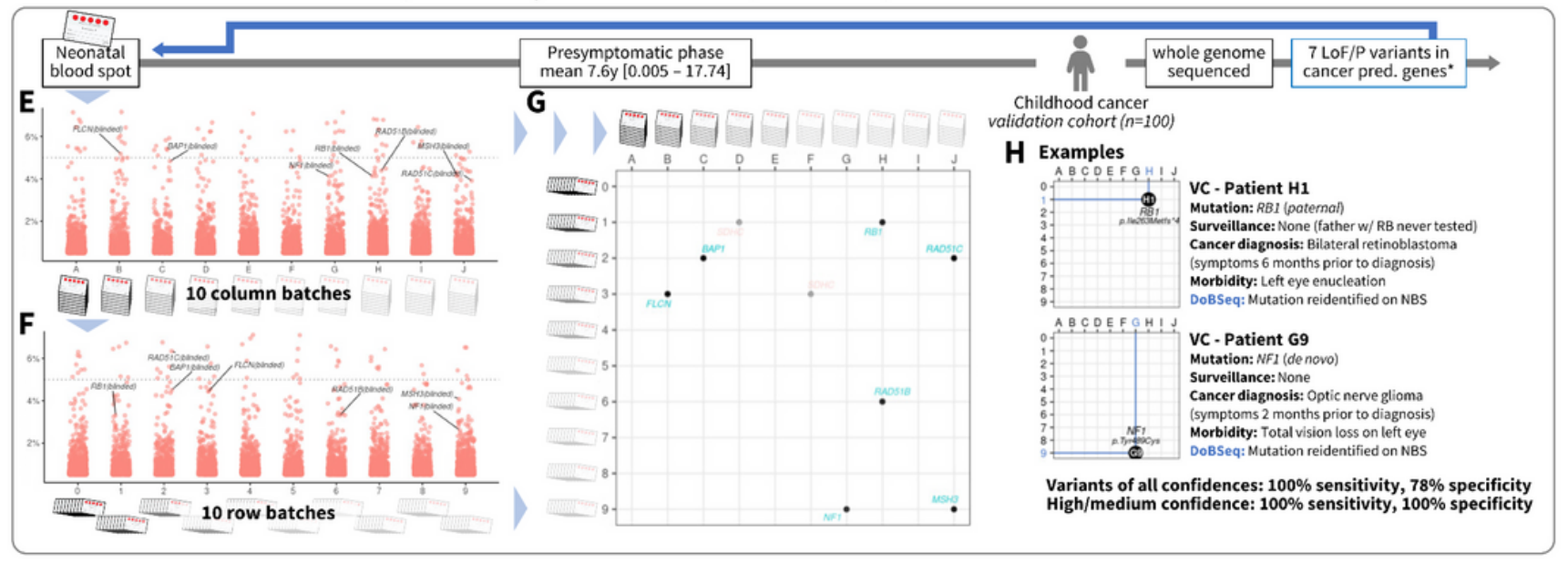

\section{Figure 1}

Illustrates the main findings and performance of double-batched sequencing (DoBSeq) in the explorative and validation cohorts. 
Upper panel: At the top; a timeline showing how all patients in the explorative cohort (EC) had neonatal blood spots (NBS) taken at birth, followed by a presymptomatic phase prior to a cancer diagnosis (equivalent to NBS sample age), after which they underwent whole genome sequencing (WGS) which identified several loss-of-function or reported pathogenic variants (LoF/P). A: Jitter plot shows LoF/P variants detected in the 10 column batches, plotted with variant allele frequency (VAF) on the $y$-axis. Blue and labeled dots represent true positive variants while red and unlabeled dots represent false positives. The gray dotted line represents the theoretically expected VAF of $5 \%$ for non-mosaic heterozygous variants (1 of 20 alleles). B: Jitter plot shows the same as A only for row batches. C: Doubly detected LoF/P variants are pinned to a specific patient in a matrix where each intersection represents one sample/patient. Dots represent DoBSeq identified variants in a gene. Teal gene names indicate true positives (found on WGS) and red gene names show false positives (not found on WGS). Higher transparency of dots/gene names indicate lower confidence. D: Showing selected patients with elaborated phenotypes, as well as DoBSeq performance compared to WGS data in the lower right corner.

Lower panel: E-H are identical to A-D, only showing data and results from the validation cohort (VC).

* The number of LoF/P variants found on whole genome sequencing refers to single nucleotide variants within the exonic regions covered by the panel used in the DoBSeq matrices. See methods for further details. 


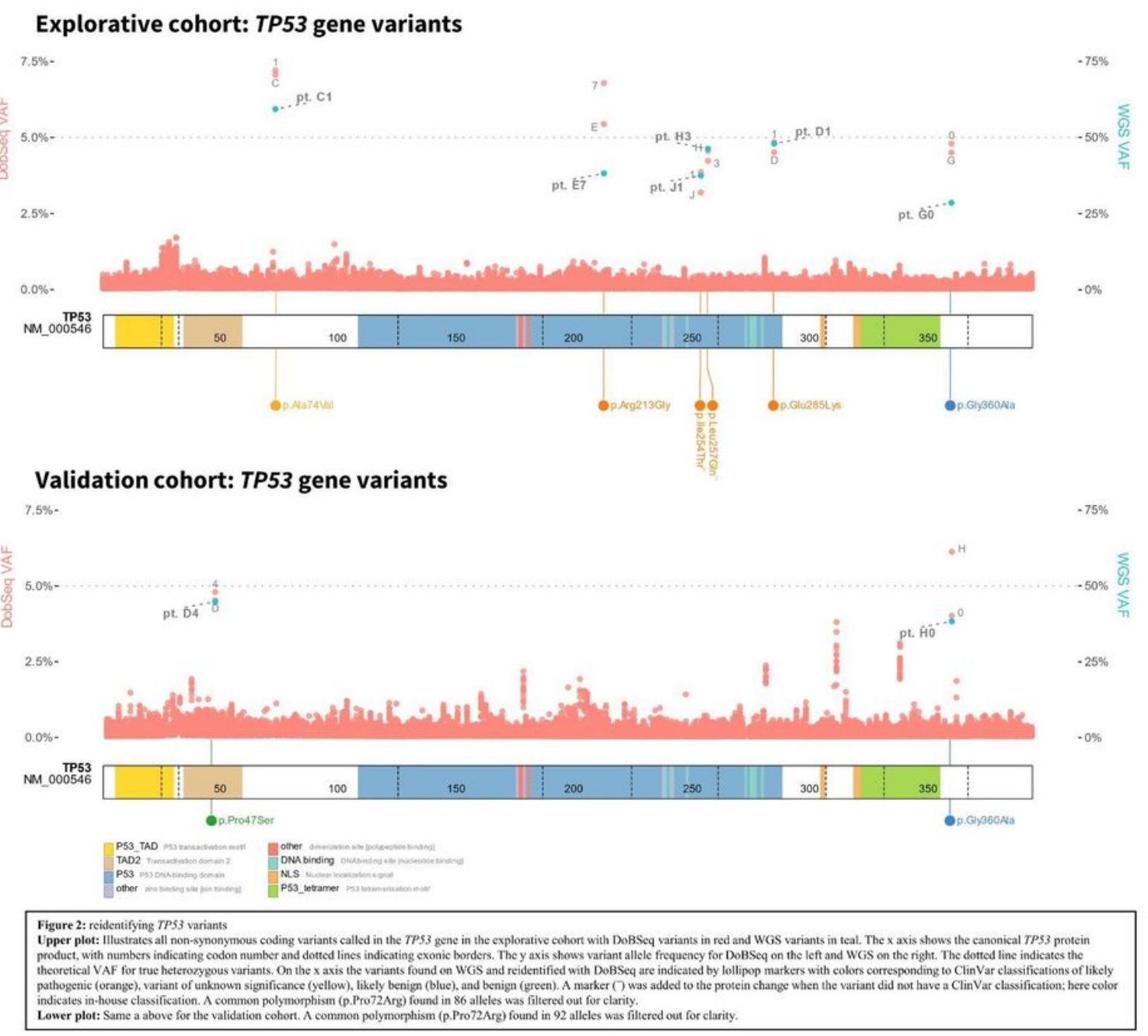

\section{Figure 2}

Reidentifying TP53 variants with double batched sequencing (DoBSeq).

Upper plot: Illustrates all non-synonymous coding variants in the TP53 gene called without any filtering, i.e. includes all low-coverage and low-confidence calls, showing DoBSeq variants found in batches from the explorative cohort (EC) in red and whole genome sequencing (WGS) variants found in the EC in teal. The $\mathrm{x}$ axis shows the canonical TP53 protein product, with numbers indicating codon number and dotted lines indicating exonic borders. The y axis shows variant allele frequency (VAF) for DoBSeq on the left and WGS on the right. The dotted line indicates the theoretical VAF for true heterozygous variants. On the $x$ axis the variants found on WGS and reidentified with DoBSeq are indicated by lollipop markers with colors corresponding to ClinVar classifications of likely pathogenic (orange), variant of unknown significance (yellow), likely benign (blue), and benign (green). A marker (") was added to the protein change when the variant did not have a ClinVar classification; here color indicates in-house classification. A common polymorphism (p.Pro72Arg) found in 86 alleles was filtered out for the sake of clarity. 
Interpretation: A steady level of sequencing noise runs along the low end of the $y$ axis with individual sets of batches rising up towards or slightly above the expected VAF. This signal allows for pin-pointing to individuals.

Lower plot: As above; for the validation cohort. A common polymorphism (p.Pro72Arg) found in 92 alleles was filtered out for the sake of clarity.
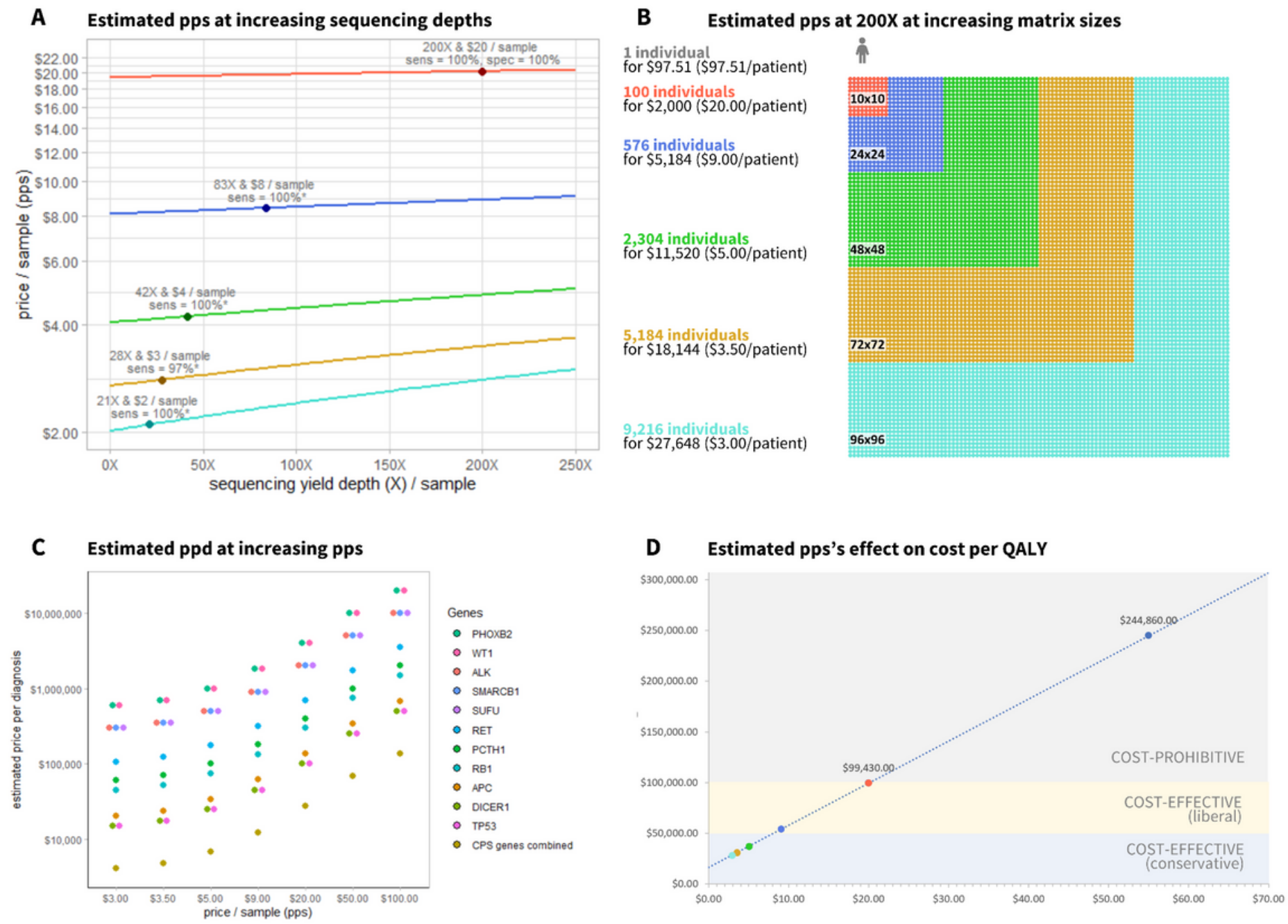

\section{Figure 3}

Illustrates scalability and estimated economic impact of price per sample (PPS).

A: The PPS stratified by the size of the matrix, with line colors corresponding to those of the text and matrices in panel 3B. The x-axis represents sequencing yield in the targeted area, under a conservative assumption of $50 \%$ quality control data loss. The logarithmic $y$-axis shows the raw estimated price of sequencing the samples from a single individual. The dots represent empirical data from single batched sequencing at the specified batch sizes, including the sensitivity for loss-of-function and/or known pathogenic (LoF/P) variant detection. 
B: Population size, total cost and PPS of individual sequencing and selected matrices at an adequate sequencing yield depth of 200X.

C: The price per diagnosis (i.e., LoFP variant detection) as influenced by PPS for 11 selected childhood cancer predisposition syndrome (CPS) genes corresponding to those investigated by Yeh et. al. ${ }^{17}$, as well as all 11 genes combined. Legend is ordered by CPS prevalence. Prevalence is based on best available evidence.

D: Based on the economic model by Yeh et al. ${ }^{17}$ this graph illustrates the cost per quality adjusted lifeyear (QALY) gained by tumor surveillance (within the 11 CPSs in panel $3 \mathrm{C}$ ) at increasing PPS. Grey, tanned, and light-blue areas represent screenings that are cost-prohibitive, cost-effective at a liberal cutoff of $\$ 100 \mathrm{k}$ per QALY, and cost-effective at a conservative cut-off of $\$ 50 \mathrm{k}$ per QALY, respectively. Colored dots correspond to PPS at selected matrix sizes (see panel 3B)

\section{Supplementary Files}

This is a list of supplementary files associated with this preprint. Click to download.

- SubmissionPREDISPOSEDPoP01032022supplemental.docx 